\title{
REGULARLY VARYING SOLUTIONS OF A LINEAR FUNCTIONAL EQUATION
}

\author{
MAREK KUCZMA
}

(Received 31 January 1974)

\begin{abstract}
We are concerned with the problem of the existence and uniqueness of regularly varying (in Karamata's sense) solutions $\varphi$ of the linear functional equation

$$
\varphi[f(x)]=\varphi(x)+h(x)
$$

in a right neighbourhood of $x=0$. Under suitable conditions on the given functions $f$ and $h$, the uniqueness of solutions depends essentially on whether the series $\Sigma h \circ f^{i}$ converges or diverges; here $f^{i}$ denotes the $i$-th functional iterate of $f$. The existence of solutions may be proved under further assumptions.
\end{abstract}

The case of the more general linear functional equation

$$
\varphi[f(x)]=g(x) \varphi(x)+h(x)
$$

may be reduced to that of equation $(*)$.

\section{Introduction}

The present paper has been inspired by Seneta (1971) who proved the existence and uniqueness of regularly varying solutions $\gamma$ for the homogeneous linear equation

$$
\gamma[f(x)]=g(x) \gamma(x) .
$$

Our purpose is to investigate to what extent those results can be generalized to the case of the inhomogeneous equation

$$
\varphi[f(x)]=g(x) \varphi(x)+h(x) .
$$

We start with preliminary results and terminology. A continuous function $F$, defined in a right vicinity $I$ of the origin and positive in a right vicinity $I_{1} \subset I$ of the origin, is said to be regularly varying whenever for every $\lambda>0$ there exists a positive and finite $\operatorname{limit}_{\lim _{x \rightarrow 0+}} F(\lambda x) / F(x)$. If this is the case, this limit is necessarily of the form 


$$
\lim _{x \rightarrow 0+} F(\lambda x) / F(x)=\lambda^{\delta},
$$

with a real constant $\delta$, and the convergence in (3) is uniform with respect to $\lambda$ in every compact interval contained in $(0, \infty)$. The number $\delta$ in (3) will be referred to as the exponent of $F$. If $\delta=0$, then $F$ is called slowly varying, and every regularly varying function $F$ has a representation

$$
F(x)=x^{8} L(x),
$$

where $\delta$ is the exponent of $F$ and $L$ is a slowly varying function. If $F$ is regularly varying, with an exponent $\delta$, then, for every $\varepsilon>0$, the inequality

$$
x^{\delta+\varepsilon}<F(x)<x^{\delta-\varepsilon}
$$

holds in a suitable vicinity of the origin. [Cf. Feller (1966), Karamata (1930), Korevaar, van Ardenne-Ehrenfest and De Bruijn (1949)].

Let $I$ denote an interval of the form $(0, a)$ or $(0, a)$, where $0<a \leqq \infty$. In the sequel we shall make the following assumptions regarding the given functions $f$, $g, h$ in equation (2).

(i) $f$ is defined, continuous and strictly increasing in $I, 0<f(x)<x$ in $I$, $f(x) / x$ is monotonic in $I$ and $s=\lim _{x \rightarrow 0+} f(x) / x \in(0,1)$.

(ii) $g$ is defined, positive, continuous and monotonic in $I$, and $g(0+)=$ $\lim _{x \rightarrow 0+} g(x) \in(0, \infty)$.

(iii) $h$ is defined and continuous in $I$ and of constant sign in a vicinity of the origin.

Write

$$
G_{n}(x)=\prod_{i=0}^{n-1} g\left[f^{i}(x)\right], \quad n=1,2, \cdots ; G_{0}(x) \equiv 1,
$$

where $f^{i}$ is the $i$-th iterate of $f$. We shall make use of the following (cf. Seneta (1971))

Theorem of Seneta. Under conditions (i) and (ii), the function

$$
\gamma_{0}(x)=\left[\lim _{n \rightarrow \infty} f^{n}(x) / f^{n}\left(x_{0}\right)\right]^{\delta_{0}} \lim _{n \rightarrow \infty} G_{n}\left(x_{0}\right) / G_{n}(x),
$$

where

$$
\delta_{0}=\log g(0+) / \log s
$$

and $x_{10}$ is arbitrarily fixed in $I$, is defined, continuous and positive in $I$, and is a regularly varying (with exponent $\delta_{0}$ ) solution of equation (1). The general regularly varying solution of (1) is given by the formula

$$
\gamma(x)=c \gamma_{0}(x),
$$

where $c$ is an arbitrary positive constant. 
Put

$$
\psi(x)=\varphi(x) / \gamma_{0}(x)
$$

Evidently $\varphi$ is regularly varying if and only if $\psi$ is regularly varying, and $\varphi$ satisfies equation (2) if and only if $\psi$ is a solution of

$$
\psi[f(x)]=\psi(x)+\frac{h(x)}{\gamma_{0}[f(x)]} .
$$

Therefore in the sequel we may restrict ouselves to the case where $g(x) \equiv 1$, i.e., we may study the equation

$$
\varphi[f(x)]=\varphi(x)+h(x)
$$

instead of (2).

\section{Uniqueness}

We have the following

LEMMA 1. Under conditions (i) and (iii), if $\varphi$ is a regularly varying solution of equation (11) in $I$, then there exists (finite or infinite) limit

$$
\lim _{x \rightarrow 0+} \varphi(x)
$$

Limit (12) is finite if and only if the series

$$
\sum_{i=0}^{\infty} h\left[f^{i}(x)\right]
$$

converges.

Proof. We need consider only the case, where $\varphi$ is a slowly varying function, for otherwise limit (12) is zero or infinity (according as the exponent of $\varphi$ is $>0$ or $<0$ ) in view of (5) applied to $\varphi$.

By iterating equation (11) we get

$$
\varphi\left[f^{n}(x)\right]=\varphi(x)+\sum_{i=0}^{n-1} h\left[f^{i}(x)\right], \quad n=1,2, \cdots
$$

Since the terms of series (13) have a constant sign (at least from some $i$ on), for every $x \in I$ there exists a finite or infinite limit

$$
\lim _{n \rightarrow \infty} \varphi\left[f^{n}(x)\right]
$$

and this limit is finite if and only if series (13) converges. So it is enough to show that, for every decreasing sequence $y_{n}$ of positive numbers, we have 


$$
\lim _{n \rightarrow \infty} \varphi\left(y_{n}\right)=\lim _{n \rightarrow \infty} \varphi\left(x_{n}\right),
$$

where $x_{n}=f^{n}\left(x_{0}\right)$, with $x_{0} \in I$ arbitrarily fixed. There is no loss of generality in assuming that $y_{0} \leqq x_{0}$.

For every $n$ there exists a $k=k(n)$ such that $y_{n} \in\left(x_{k+1}, x_{k}\right]$. We put $x_{n}^{*}=x_{k(n)}$. Then $x_{n}^{*}$ is a subsequence of $x_{n}$, possibly with repetitions of terms; in any case

$$
\lim _{n \rightarrow \infty} \varphi\left(x_{n}^{*}\right)=\lim _{n \rightarrow \infty} \varphi\left(x_{n}\right) .
$$

Fix $s^{\prime}, 0<s^{\prime}<s$. Since $\varphi$ is slowly varying, we have

$$
\lim _{x \rightarrow 0+} \varphi(\lambda x) / \varphi(x)=1
$$

uniformly in $\left[s^{\prime}, 1\right]$. On the other hand, we have for sufficiently large $n$

$$
s^{\prime} \leqq \frac{f\left(x_{n}^{*}\right)}{x_{n}^{*}}<\frac{y_{n}}{x_{n}^{*}} \leqq 1,
$$

whence

$$
\text { . } \lim _{n \rightarrow \infty} \varphi\left(y_{n}\right) / \varphi\left(x_{n}^{*}\right)=1 .
$$

Now (15) results from (16) and (17).

It may be worth while to note that, without any further assumptions, a slowly varying function need not approach a definite limit as $x \rightarrow 0+$, as may be seen from the example of the function $\varphi(x)=\exp \sin \log \log x^{-1}$.

TheOREM 1. Under conditions (i) and (iii), if series (13) converges, then equation (11) may have at most a one-parameter family of regularly varying solutions $\varphi$. These solutions, when they exist, are given by the formula

$$
\varphi(x)=C-\sum_{i=0}^{\infty} h\left[f^{i}(x)\right], \quad C \geqq 0 .
$$

Proof. Let $\varphi$ be a regularly varying solution of (11). By Lemma 1 there exists a finite limit

$$
C=\lim _{x \rightarrow 0+} \varphi(x) \geqq 0 .
$$

Letting $n \rightarrow \infty$ in formula (14) and taking (19) into account we obtain (18).

The situation is different in the case where series (13) diverges. Namely, we have the following

THEOREM 2. Under conditions (i) and (iii), if series (13) diverges, then either 
equation (11) has no regularly varying solution, or every continuous positive solution of equation (11) is regularly varying (with the same exponent).

Proof. Let $\varphi_{0}$ be a regularly varying solution of equation (11). By Lemma 1

$$
\lim _{x \rightarrow 0+} \varphi_{0}(x)=+\infty .
$$

Let $\varphi$ be an arbitrary continuous positive solution of equation (11). Then the function $\alpha=\varphi-\varphi_{0}$ is a continuous solution of the equation

$$
\alpha[f(x)]=\alpha(x),
$$

and hence $\alpha$ is bounded in $I$. Consequently

$$
\lim _{x \rightarrow 0+} \frac{\varphi(\lambda x)}{\varphi(x)}=\lim _{x \rightarrow 0+} \frac{\varphi_{0}(\lambda x)+\alpha(\lambda x)}{\varphi_{0}(x)+\alpha(x)}=\lim _{x \rightarrow 0+} \frac{\varphi_{0}(\lambda x)}{\varphi_{0}(x)},
$$

i.e., $\varphi$ is regularly varying with the same exponent as $\varphi_{0}$.

Let us note that the general continuous solution of equation (11) depends on an arbitrary function [cf. Kuczma (1968)]. Therefore it seems that in the case where series (13) diverges the class of asymptotically regular functions is better suited for equation (11) than that of regularly varying functions [cf. Coifman and Kuczma (1969)].

Of course, in general it may happen that series (13) converges for some values of $x$ in $I$, and diverges for other; but it follows from Lemma 1 that in such a case equation (11) cannot have regularly varying solutions.

\section{Existence}

If series (13) converges uniformly on every compact subinterval of $I$, then [and only then; cf. Kuczma (to appear)] functions (18) actually fulfil condition (19). It follows that for $C>0$ functions (18) are slowly varying. However, for $C=0$ it is necessary to make stronger assumptions to ensure that function (18) is regularly varying.

Lemma 2. If $f$ fulfils condition ( $i$ ), and $h$, defined, continuous and positive in $I$, is a regularly varying function with an exponent $\delta>0$, then the function

$$
\Phi(x)=\sum_{i=0}^{\infty} h\left[f^{i}(x)\right]
$$

also is regularly varying with the exponent $\delta$.

Proof. Take an arbitrary $\varepsilon>0$ such that $\varepsilon<\min (s, 1-s)$ and

$$
s_{1}=(s+\varepsilon)^{\delta}(1+\varepsilon)<1 \text {. }
$$


Write

$$
s_{2}=(s-\varepsilon)^{\delta}(1-\varepsilon)
$$

and $L(x)=x^{-\delta} h(x)$ so that $L$ is a slowly varying function. Lastly, fix a positive $\mu<\delta$.

We may find an $A>0$ such that the inequalities

$$
\begin{gathered}
s-\varepsilon<f(x) / x<s+\varepsilon, \\
1-\varepsilon<L[f(x)] / L(x)<1+\varepsilon, \\
0<h(x)<x^{\mu},
\end{gathered}
$$

hold for $x \in(0, A)$ (cf., in particular, (5)). It follows from (20) and (22) that series (13) converges uniformly on every compact subinterval of $I$. Consequently $\Phi$ is a continuous and positive function in $I$.

Relations (20) and (21) imply that

$$
\begin{gathered}
(s-\varepsilon)^{i}<\frac{f^{i}(x)}{x}<(s+\varepsilon)^{i} \text { and }(1-\varepsilon)^{i}<\frac{L\left[f^{i}(x)\right]}{L(x)}<(1+\varepsilon)^{i} \\
\text { for } \quad x \in(0, A) \text { and } i=1,2, \ldots
\end{gathered}
$$

Since for $x \in(0, A / \lambda)$ we have $\lambda x \in(0, A)$, we get hence for $x \in(0, A / \lambda)$ and $i=1,2, \ldots$

$$
\lambda(s-\varepsilon)^{i}<\frac{f^{i}(\lambda x)}{x}<\lambda(s+\varepsilon)^{i}
$$

and

$$
\frac{L(\lambda x)}{L(x)}(1-\varepsilon)^{i}<\frac{L\left[f^{i}(\lambda x)\right]}{L(x)}<\frac{L(\lambda x)}{L(x)}(1+\varepsilon)^{i} .
$$

Thus we obtain the estimation, valid for $x \in(0, A / \lambda)$,

$$
\begin{aligned}
\frac{\Phi(\lambda x)}{h(x)} & =\frac{h(\lambda x)}{h(x)}+\sum_{i=1}^{\infty} \frac{h\left[f^{i}(\lambda x)\right]}{h(x)} \\
& =\frac{h(\lambda x)}{h(x)}+\sum_{i=1}^{\infty}\left(\frac{f^{i}(\lambda x)}{x}\right)^{\delta} \frac{L\left[f^{i}(\lambda x)\right]}{L(x)} \\
& <\frac{h(\lambda x)}{h(x)}+\sum_{i=1}^{\infty} \lambda^{\delta}(s+\varepsilon)^{i \delta} \frac{L(\lambda x)}{L(x)}(1+\varepsilon)^{i} \\
& =\frac{h(\lambda x)}{h(x)}+\lambda^{\delta} \frac{L(\lambda x)}{L(x)} \sum_{i=1}^{\infty} s_{1}^{i} \\
& =\lambda^{\delta} \frac{L(\lambda x)}{L(x)}\left(1+\sum_{i=1}^{\infty} s_{1}^{i}\right)=\lambda^{\delta} \frac{L(\lambda x)}{L(x)} \frac{1}{1-s_{1}},
\end{aligned}
$$

and similarly, for $x \in(0, A / \lambda)$, 


$$
\frac{\Phi(\lambda x)}{h(x)}>\lambda^{s} \frac{L(\lambda x)}{L(x)} \frac{1}{1-s_{2}} .
$$

Hence we obtain on letting $x \rightarrow 0+$

$$
\lambda^{\delta} \frac{1}{1-s_{2}} \leqq \liminf _{x \rightarrow 0+} \frac{\Phi(\lambda x)}{h(x)} \leqq \limsup _{x \rightarrow 0+} \frac{\Phi(\lambda x)}{h(x)} \leqq \lambda^{\delta} \frac{1}{1-s_{1}} .
$$

Now we let $\varepsilon \rightarrow 0$ and we get by (23)

$$
\lim _{x \rightarrow 0+} \frac{\Phi(\lambda x)}{(x)}=\frac{\lambda^{\delta}}{1-s^{\delta}} .
$$

(24) is valid for every $\lambda>0$; in particular, for $\lambda=1$ (24) becomes

$$
\lim _{x \rightarrow 0+} \frac{\Phi(x)}{h(x)}=\frac{1}{1-s^{\delta}}
$$

Relations (24) and (25) imply that

$$
\lim _{x \rightarrow 0+} \frac{\Phi(\lambda x)}{\Phi(x)}=\lambda^{\delta}
$$

which was to be proved.

As an immediate consequence of Theorem 1 and Lemma 2 and of the remarks preceding Lemma 2 , we obtain the following

THEOREM 3. Suppose that $f$ fulfils condition (i), and $h$, defined, continuous and negative in $I$, is such that - $h$ is a regularly varying function with an exponent $\delta>0$. Then equation (11) has exactly one-parameter family of regularly varying solutions. These solutions are given by formula (18) and are slowly varying for $C>0$, and regularly varying with the exponent $\delta$ for $C=0$.

For the case where series (13) diverges we have the following simple result.

THEOREM 4. Suppose that $f$ and $h$ fulfil conditions (i) and (iii) and, moreover, $h$ is monotonic in I and the limit $\lim _{x \rightarrow 0+} h(x)$ is finite and positive. Then every continuous and positive solution $\varphi$ of equation (11) in I is slowly varying.

Proof. Since $\lim _{x \rightarrow 0+} h(x) \neq 0$, series (13) diverges and so, in view of Theorem 2, it is enough to show that equation (11) has at least one slowly varying solution.

Set $\tilde{g}(x)=\exp h(x)$. This function fulfils condition (ii) and

$$
\lim _{x \rightarrow 0+} \tilde{g}(x)>1
$$

By the Theorem of Seneta the equation

$$
\tilde{\gamma}[f(x)]=\tilde{g}(x) \tilde{\gamma}(x)
$$


has a regularly varying solution $\tilde{\gamma}_{0}(x)$, and it follows by (26) that $\lim _{x \rightarrow 0+} \tilde{\gamma}_{0}(x)=$ $\infty$. Consequently the function

$$
\varphi_{0}(x)=\log \tilde{\gamma}_{0}(x)
$$

is a slowly varying solution of equation (11).

\section{General linear equation}

Below we translate our Theorems $1-3$ to the case of the general linear equation (2). We obtain these results applying Theorems $1-3$ to equation (10), where $\psi$ is defined by (9) and $\gamma_{0}$ by (7). Theorem 4 has no such a readily available analogue for equation (2).

In the theorems below $G_{i}$ are given by (6) and $\delta_{0}$ by (8).

THEOREM 1'. Under conditions (i), (ii), and (iii), if the series

$$
\sum_{i=0}^{\infty} \frac{h\left[f^{i}(x)\right]}{G_{i+1}(x)}
$$

converges, then equation (2) may have at most a one-parameter family of regularly varying solutions $\varphi$. These solutions, when they exist, are given by the formula

$$
\varphi(x)=C \gamma_{0}(x)-\sum_{i=0}^{\infty} \frac{h\left[f^{i}(x)\right]}{G_{i+1}(x)}, \quad C \geqq 0 .
$$

THEOREM 2'. Under conditions (i), (ii), and (iii), if series (27) diverges, then either equation (2) has no regularly varying solution, or every continuous positive solution of equation (2) is regularly varying (with the same exponent).

Theorem 3'. Suppose that $f$ and $g$ fulfil conditions (i) and (ii), and $h$, defined continuous and negative in $I$, is such that $-h$ is a regularly varying function with an exponent $\delta>\delta_{0}$. Then equation (2) has exactly one-parameter family of regularly varying solutions. These solutions are given by formula (28) and for $C>0$ have the exponent $\delta_{0}$, whereas for $C=0$ the exponent $\delta$.

\section{References}

R. R. Coifman and M. Kuczma (1969), 'On asymptotically regular solutions of a linear functional equation', Aequationes Math. 2, 332-336.

W. Feller (1966), 'An Introduction to Probability Theory and its Applications', Vol. 2, John Wiley \& Sons, New York.

J. Karamata (1930), 'Sur une mode de croissance regulière', Mathematica Cluj 4, 38-53.

J. Korevaar, T. van Ardenne-Ehrenfest and N. G. De Bruijn (1949), 'A note on slowly oscillating functions', Nieuw Archief voor Wiskunde 23, 77-86. 
M. Kuczma (1968), 'Functional Equations in a Single Variable', Monografie Mat. 46, Polish Scientific Publishers, Warszawa.

M. Kuczma (to appear), 'On some properties of solutions of a functional equation'.

E. Seneta (1971), 'On invariant measures for simple branching process', J. Appl. Probability 8, 43-51.

\section{Mathematics Department}

Silesian University

Katowice, Poland. 